\title{
Correction to: Research on Video Violence Detection Technology of UAV on Cloud Platform
}

Chen Zhi and Weidong Bao

\author{
Correction to: \\ Chapter "Research on Video Violence Detection Technology \\ of UAV on Cloud Platform" in: X. Sun et al. (Eds.): \\ Artificial Intelligence and Security, CCIS 1252, \\ https://doi.org/10.1007/978-981-15-8083-3_33
}

In the originally published version of chapter 33, the name of one of the authors was incorrect. The author name has been changed to Chen Zhi. 